\title{
Optimización de señal de control en reguladores PID con arquitectura antireset Wind-Up
}

\section{Control signal optimization for PID regulators based on a Wind-Up anti-reset architecture}

\section{Ilber Adonayt Ruge Ruge}

Ingeniero electrónico, magíster en Ingeniería de Control Industrial. Docente e investigador de la Universidad de Cundinamarca. Fusagasugá, Colombia.iruge@ mail.unicundi.edu.co

Fecha de recepción: 26 de febrero de 2011

Clasificación del artículo: Investigación (Conciencias)

Fecha de aceptación: 30 de mayo de 2011

Palabras clave: Antireset Wind-Up, control, evaluación de desempeño, optimización, PID.

Key words: Anti-reset Wind-Up, control, performance evaluation, optimization, PID.

\section{RESUMEN}

El presente artículo muestra al lector los métodos de sintonización de controladores PID Kayser-Rajka (KR) y Astrom-Haglund (AH), con el ánimo de evaluar su desempeño frente a algunos métodos convencionales de sintonización como Ziegler-Nichols (ZN). Además, se muestra el método de mejoramiento de la señal de control basado en la arquitectura Antireset Wind-Up.

\section{ABSTRACT}

This paper shows the reader the methods of tuning PID controllers Kayser-Rajka (KR) and Astrom-Haglund ( $\mathrm{AH})$, with the aim of evaluating their performance against some conventional methods like Ziegler-Nichols tuning (ZN). It also shows the method for improving the control signal based on the architecture Antireset Wind-Up.

\section{INTRODUCCIÓN}

La sintonización de los controladores PID consiste en la determinación del ajuste de sus parámetros $(K c, T i, T d)$, para lograr un comportamiento del sistema de control aceptable y robusto de conformidad con algún criterio de desempeño establecido.

Para poder realizar la sintonización de los controladores, primero se debe identificar la dinámica del proceso, y a partir de ésta determinar los pa- 


\section{con-ciencias}

rámetros del controlador utilizando el método de sintonización seleccionado.

En la práctica suele aparecer el fenómeno de resetwind-up al arrancar el sistema o en cualquier otra situación en la que surge un error grande durante un tiempo prolongado. Sucede, entonces, que el integrador aumenta su salida para reducir el error aun cuando la señal de control esté saturada. Esto hace que cuando el sistema se acerque al punto de referencia el término integral haya crecido y se sobrepase el valor deseado, ya que la única forma de reducir el valor del término integral es mediante un cambio de signo a su entrada (señal de error).

Para evitar este fenómeno existe un algoritmo denominado anti resetwind-up. El propósito del artículo es mostrar las bondades de este algoritmo frente a las técnicas convencionales de controladores como el PID.

\section{METODOLOGÍA}

Para la evaluación de desempeño del controlador PID con optimización de señal de control con arquitectura antireset wind-up, se optó por diseñar inicialmente el regulador PID mediante las técnicas de sintonización de Ziegler-Nichols en lazo abierto y lazo cerrado, Kayser-Rajka y AstromHaglund. Esto con el propósito de seleccionar el controlador que ofrezca la mejor respuesta ante una entrada escalón unitario como señal de prueba del regulador y poder comparar el desempeño del controlador con respecto a la incorporación de la arquitectura antireset wind-up para optimización de la señal de control.

\subsection{Sintonización de controlador mediante método de Ziegler-Nichols en lazo abierto}

Para este método [1 - 3] se debe aplicar una señal escalón unitario a la entrada del sistema a controlar, y determinar los parámetros K, L y T según se muestra en la Fig. 2.

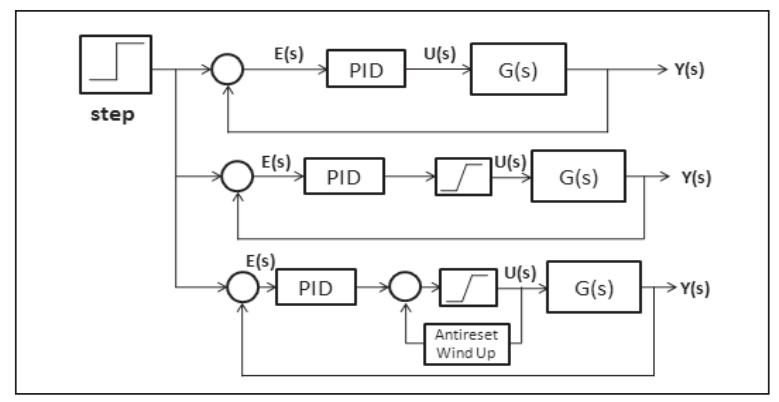

Fig. 1. Metodología basada en comparación de desempeño para evaluación de controlador con antireset wind-up.

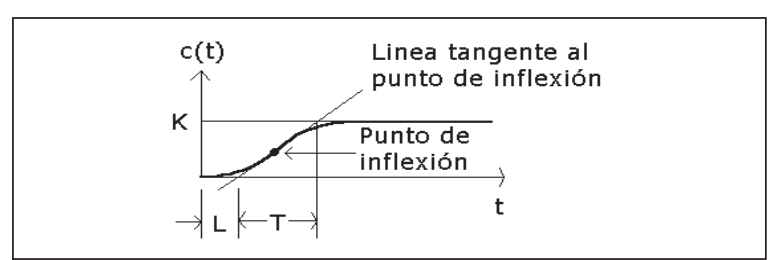

Fig. 2. Parámetros para sintonización de controlador mediante $\mathrm{ZN}$ en lazo abierto

La función de transferencia modelo utilizada para la sintonización del controlador PID, mediante las técnicas mencionadas es:

$$
G(s)=\frac{1}{(s+1)^{3}}
$$

La respuesta ante una entrada escalón unitario es:

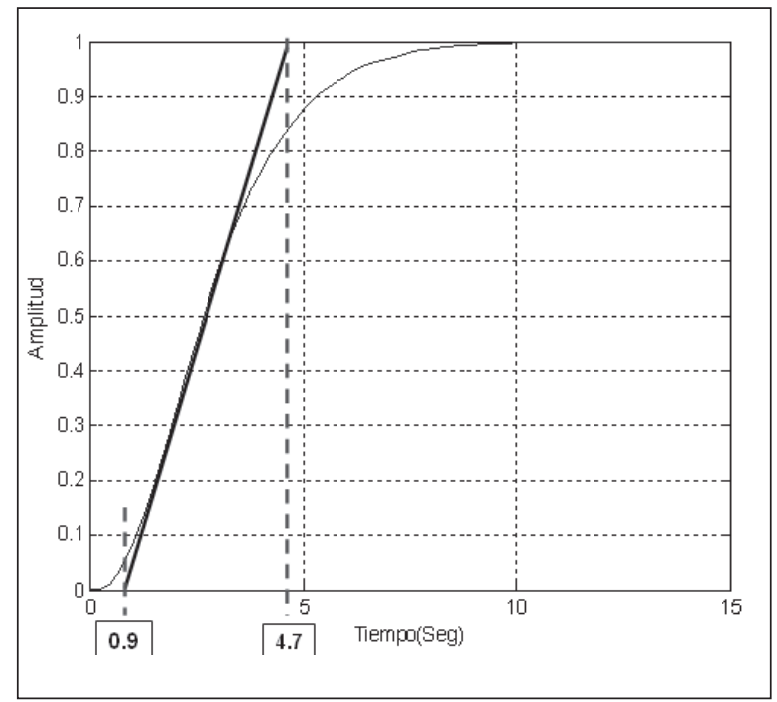

Fig. 3. Respuesta del sistema G(s) ante entrada escalón unitario y medida de parámetros $\mathrm{K}, \mathrm{L}$ y T. 


\section{con-ciencias}

De la Fig. 3 se tiene:

$$
\begin{gathered}
K=1 \\
L=0,9 s \\
T=3,8 s
\end{gathered}
$$

Para calcular las constantes Kp Ti y Td del controlador PID, se utilizan las fórmulas mostradas en la tabla 1.

Tabla 1. Cálculo de parámetros mediante $\mathrm{ZN}$ en lazo abierto.

\begin{tabular}{|c|c|c|c|}
\hline Tipo & Kp & Ti & Td \\
\hline $\mathrm{P}$ & $\mathrm{T} / \mathrm{L}$ & inf & 0 \\
\hline $\mathrm{PI}$ & $0,9(\mathrm{~T} / \mathrm{L})$ & $\mathrm{L} / 0,3$ & 0 \\
\hline $\mathrm{PID}$ & $1,2(\mathrm{~T} / \mathrm{L})$ & $2 \mathrm{~L}$ & $0,5 \mathrm{~L}$ \\
\hline
\end{tabular}

Reemplazando:

$$
\begin{gathered}
K p=\frac{1,2}{T L}=4,22 \\
K i=\frac{K p}{T i}=\frac{K p}{2 L}=2,34 \\
K d=K p \cdot T d=K \mathrm{p}(0,5 \mathrm{~L})=1,89
\end{gathered}
$$

\subsection{Sintonización de controlador mediante método de Ziegler-Nichols en lazo cerrado}

Para este método [1 - 3] se aplica una señal escalón unitario a la entrada, se realimenta el lazo y se determina el valor de la ganancia $(\mathrm{Kp})$ que hace al sistema críticamente estable y se mide el periodo de salida $(\mathrm{Pcr})$.

Se utiliza Matlab [3], [4] como herramienta de simulación para establecer la ganancia crítica del sistema $\mathrm{G}(\mathrm{s})$.

Para calcular las constantes $K p$, Ti y Td del controlador PID, se utilizan las fórmulas mostradas en la tabla 2. 


\section{con-ciencias}

Reemplazando:

$$
\begin{gathered}
K p=0,6^{*} K c r=4,8 \\
K i=\frac{K p}{T i}=\frac{K p}{0,5^{*} P c r}=2,64 \\
K d=K p \cdot T d=K \mathrm{p}\left(0,125^{*} \mathrm{Pcr}\right)=2,17 \\
K d=K p \cdot T d=K \mathrm{p}\left(0,125^{*} \mathrm{Pcr}\right)=2,17
\end{gathered}
$$

\subsection{Sintonización de controlador mediante método de Astrom-Haglund}

Este método [5] - [7] incorpora un relé (Relay) que provoca oscilaciones controladas en el proceso que permiten la identificación de características dinámicas del mismo.

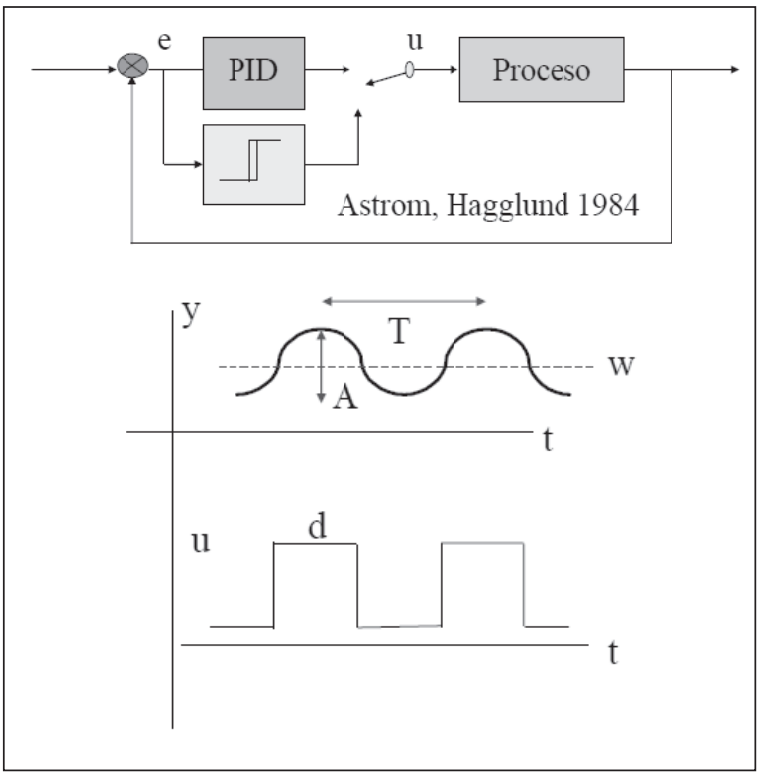

Fig. 6. Método de sintonización Astrom-Haglund.

Se utiliza Matlab como herramienta de simulación para establecer los parámetros A y T del sistema $\mathrm{G}(\mathrm{s})$ al introducir el relé con amplitud $\boldsymbol{d}$ dentro del lazo cerrado.

Para calcular las constantes Kp Ti y Td del controlador PID, se mide la amplitud de la oscilación de salida $(a=0,31)$ y el periodo del mismo
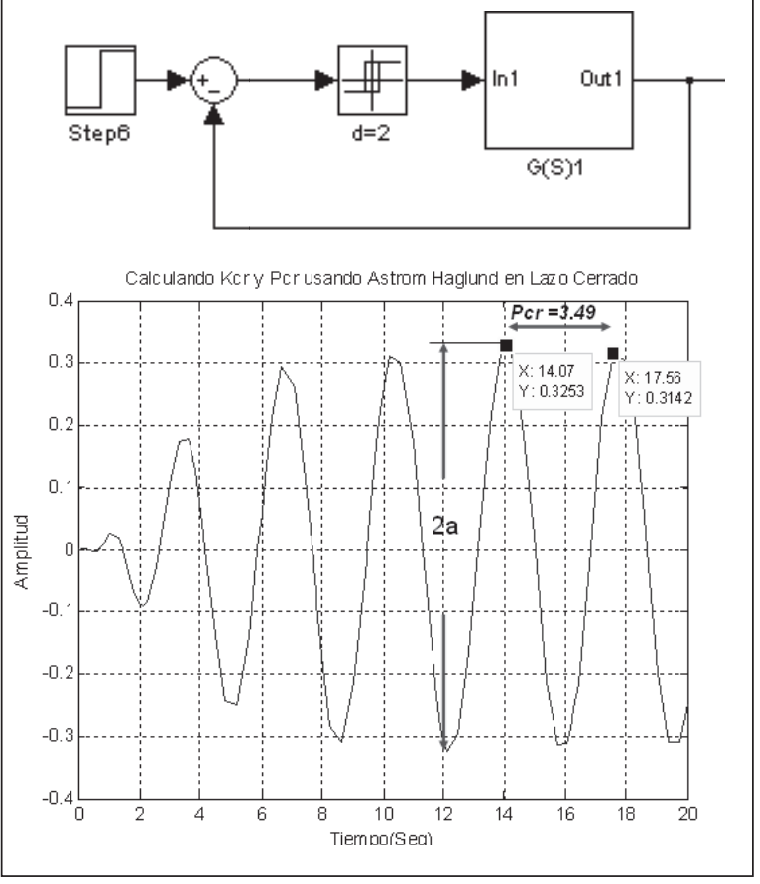

Fig. 7. Método de Astrom-Haglund con amplitud de relé $\mathrm{d}=2$.

$(P c r=3,49)$. Se determina el valor de $\mathrm{Kcr}$ dado por la siguiente ecuación:

$$
K c r=\frac{4 d}{\pi a}
$$

Después se utilizan las fórmulas usadas para Ziegler-Nichols en lazo cerrado de la tabla 2. Reemplazando:

$$
\begin{gathered}
K p=0,6 K c r=4,92 \\
K i=\frac{K p}{T i}=\frac{K p}{0,5 P c r}=2,81 \\
K d=K p(0,125 P c r)=2,14
\end{gathered}
$$

\subsection{Sintonización de controlador mediante método de Kayser-Rajka}

Este método trabaja similar al método de $\mathrm{AH}$, solo que incorpora un retardo (Transport Delay) después del relé (Relay). Esto también provoca 


\section{con-ciencias}


Fig. 8. Método de Kayser-Rajka.

oscilaciones controladas en el proceso que permiten la identificación de características dinámicas del proceso.

Para este método se busca obtener una oscilación controlada de salida, proporcional a los valores de amplitud del relé $d$ y el valor del retardo $T d$, para que se cumpla el siguiente criterio de diseño:

$$
\Phi=\frac{360 \cdot T d}{T c}
$$

Donde $T c$ es el periodo de oscilación de salida del sistema.

Para un valor de $\Phi=10$, se debe encontrar el valor de $T d$ (Transport Delay) para que cumpla el criterio dado en (3), es decir que:

$$
T c=36 * T d
$$

Si d=2 y Td=0,12 (aprox. 4,3/36), de la Fig. 7 se puede obtener que:

$$
\mathrm{Tc}=4,30
$$

Cumpliendo el criterio deseado en (4). De la Fig. 7, el valor para la amplitud de oscilación de salida (a) es:

$$
\mathrm{a}=0,43
$$

Ahora, se calcula el valor de $K c r$ :

$$
K c r=\frac{4 d}{\mathrm{p} a}=5,92
$$

Se utilizan las fórmulas de la tabla 2 para determinar los parámetros $K p$, Ki y $K d$ del controlador PID.

$$
\begin{gathered}
K p=0,6^{*} K c r=3,55 \\
K i=\frac{K p}{T i}=\frac{K p}{0,5^{*} P c r}=1,65 \\
K d=K p \cdot T d=K p\left(0,125^{*} \mathrm{Pcr}\right)=1,90
\end{gathered}
$$

\section{RESULTADOS}

La Fig. 9 muestra la comparación entre los controladores $\mathrm{ZN}$ en lazo abierto, ZN en lazo cerrado, Astrom-Haglund y Kayser-Rajka.

Del resultado mostrado en la Fig.9, se observa que la respuesta debida al control PID sintonizado por el método de Kayser-Rajka $(K R)$, ofrece el menor factor de amortiguación (aprox. 32\%) y el menor tiempo de estabilización (aprox. 5,5s) con respecto a la respuesta dada por los otros controladores.

\subsection{Sistema anti Wind-Up frente a técnica de saturación}

En todos los sistemas de control, los actuadores y las señales de salida de los controladores tienen un rango limitado de operación [5]. Una señal de con- 




Fig. 9. Comparación de resultados de los 4 métodos de sintonización discutidos.

trol fuerte disminuye la vida útil de los actuadores del proceso. El método convencional es la adición de saturadores o limitadores a la salida de los controladores para disminuir este efecto nocivo.

\subsection{Sistema Antireset Wind-Up}

Este sistema consiste básicamente en la saturación del término integral del controlador PID, para impedir el fenómeno de generación de señales de control amplias, debidas a cambios bruscos y grandes de la señal de error [5].

\subsection{Arquitectura sistema AntiReset Wind-Up}

El sistema consiste en el seguimiento al comportamiento de la señal de control para ajustar el tér-



Fig. 10. Limitación de señal de control mediante saturadores.

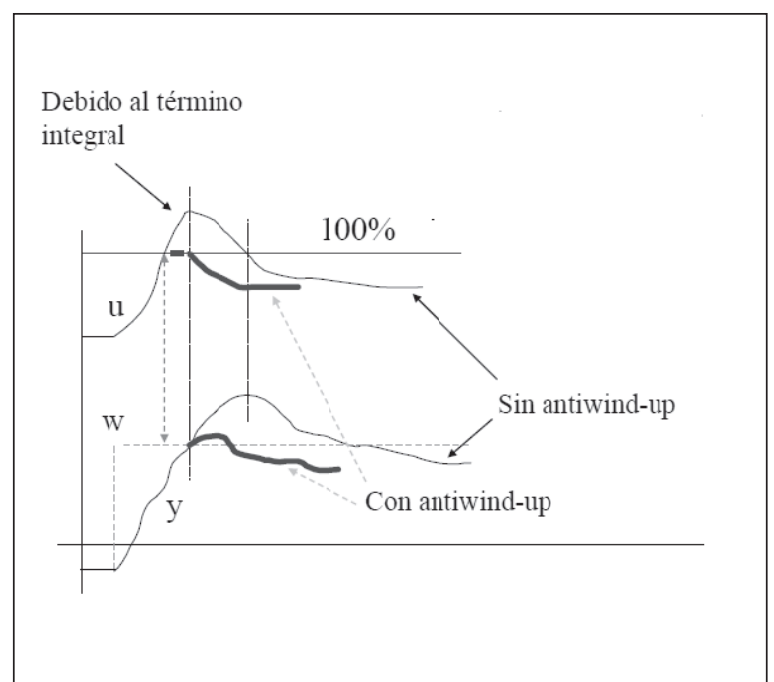

Fig. 11. Efecto del sistema Anti Reset Wind-Up.

mino integrativo del controlador ( $\mathrm{si} \mathrm{v}>\mathrm{u}$ corregir término integral hasta que $\mathrm{v}=\mathrm{u}$ ).



Fig. 12. Arquitectura sistema Anti reset Wind-Up.

Para evaluar el comportamiento de este sistema, frente al resultado que se obtiene utilizando los sistemas de saturación clásica y sin saturación, se toma como referencia el sistema de control obtenido mediante el método de sintonización de $\mathrm{ZN}$ en lazo cerrado, como método clásico analizado en el capítulo VI. La implementación de este sistema usando Simulink de Matlab se muestra en el Anexo A. En esta arquitectura se adiciona el efecto de la variación de la salida al bloque controlador (derivada de la salida) [3] y los valores de las constantes $\mathrm{Kp}$, Ki y Kd del controlador PID son los obtenidos mediante método de $\mathrm{ZN}$ en lazo cerrado. 


\section{con-ciencias}

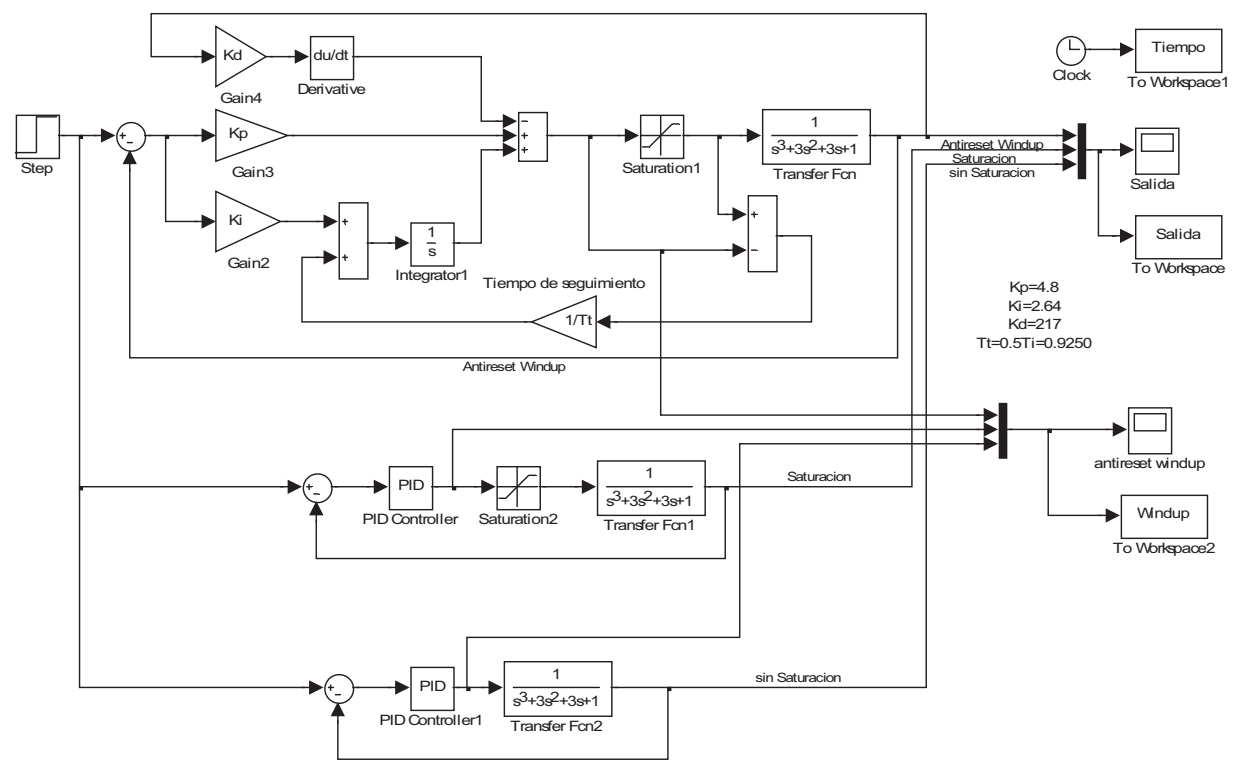

Fig. 13. Sistema Anti reset Wind-Up vs Sistema de saturación

Para una señal de entrada escalón unitario, la Fig. 14 muestra el efecto de la arquitectura anti reset wind-up sobre la señal de control U(s) del sistema de control realimentado.

Es claro apreciar las ventajas de utilizar el sistema anti reset wind-up, ya que permite "suavizar" la señal de control, es decir, amortigua el sobrepico

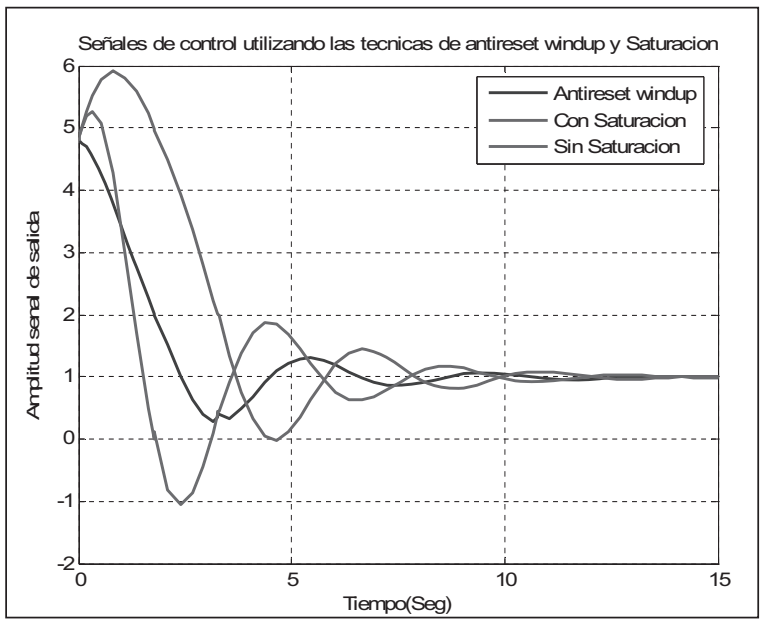

Fig. 14. Resultado de adición del Anti Reset Wind-Up en sistema de control. debido al cambio de la señal de referencia (señal escalón). Lo anterior es una característica práctica deseable, puesto que aumenta la vida útil del actuador en la planta.

Los resultados de la Fig. 15 muestran que el sistema anti reset wind-up, además de mejorar las condiciones de la señal de control, también

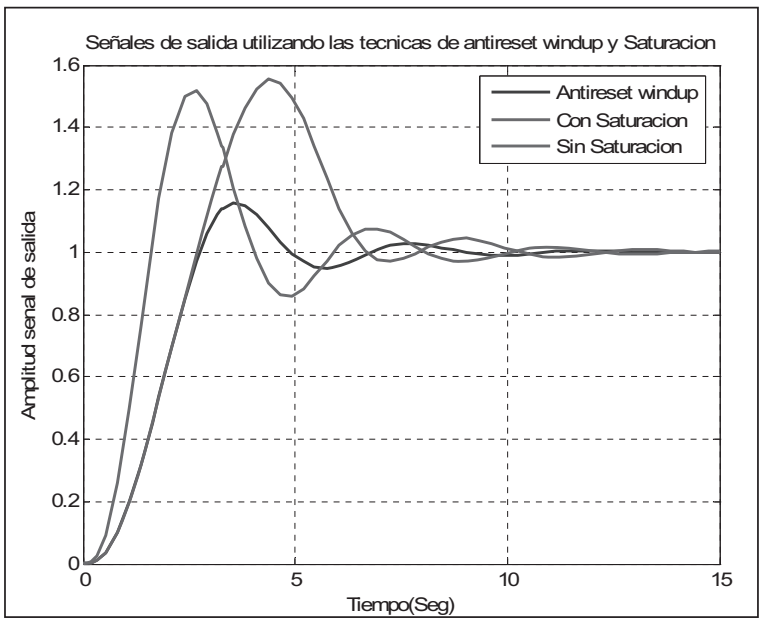

Fig. 15. Resultado de la adición del Anti Reset WindUp en sistema de control. 
mejora el sobre amortiguamiento de la señal de salida, pasando de un sobre amortiguamiento del $50 \%$ con respecto al sistema sin saturación a un $18 \%$.

Otro factor importante para resaltar, es que el tiempo de establecimiento (Setting Time) se mantiene constante para los tres casos analizados (anti reset wind-up, con saturación y sin saturación).

\section{CONCLUSIONES}

Los sistemas de sintonización de controladores PID como Ziegler-Nichols basan su principio de sintonización en la obtención de la ganancia crítica que hace que el sistema en lazo cerrado oscile, pero cuando el sistema en lazo cerrado es estable para todo Kc este sistema no es aplicable. Es aquí donde técnicas como Kayser-Rajka y Astrom-Haglund cobran interés, dado su característica de incorporar oscilaciones controladas por medio de elementos conmutadores (relay) que también permiten obtener de mane- ra eficiente los parámetros de sintonización del controlador PID.

De acuerdo con los resultados obtenidos con la incorporación del sistema anti wind-up en el lazo de control, la desventaja es que el tiempo de subida (Rise Time) aumenta con respecto a la respuesta del sistema sin limitación. Pero este fenómeno es claro cuando se diseñan controladores, puesto que si se desea mejorar el factor de amortiguamiento, indiscutiblemente se tendrá que sacrificar un poco el tiempo de subida y viceversa.

El efecto más importante de incorporar el sistema anti wind-up en este sistema de control, fue que al mejorar las características de la señal de control; haciéndola menos nociva para los actuadores del sistema, también mejoró el factor de amortiguamiento de la señal de salida. Además, el tiempo de establecimiento se mantiene constante, lo que permite decidir que el comportamiento del sistema anti reset wind-up es adecuado para ser utilizado en la implementación de sistemas de control en áreas de aplicación industrial.

\section{REFERENCIAS}

[1] K. Ogata, Ingeniería de Control Moderna: Reglas de Sintonía de Controladores PID, Madrid: Prentice Hall, 1996.

[2] B. Kuo, Sistemas de Control Automático, Décima Edición, México: Prentice Hall, 2004.

[3] R. Dorf, Sistemas de Control Moderno, Décima Edición, España: Prentice Hall, 2009.

[4] User's Guide. Version 4.2., System Control Toolbox for use with Matlab user guide, 2010. [En línea]. Disponible en: http:// www-lar.deis.unibo.it/ lmarconi/files/control_tb.pdf.

[5] J. de la Fuente, "Sintonía de reguladores PID," Facultad de Ciencias, Universidad de Valladolid .2010. [En línea]. Disponible en: http://www.isa.cie.uva.es/ maria/pids.pdf

[6] V. Alfaro, "Métodos de sintonización de controladores PID que operan como reguladores," Ingeniería 12, vol. 1, no. 2, pp. Oct. 2002 [En línea]. Disponible en: http://eie. ucr.ac.cr/uploads/file/documentos/pub_inv/ articulos/valfaro02B.pdf. 